\title{
Effects of Proton Pump Inhibitor Therapy in the Distal Gut: Putting the Pieces Together
}

\author{
Daniel Keszthelyi • Ad A. Masclee
}

Received: 19 July 2012/ Accepted: 24 July 2012/Published online: 17 August 2012

(C) Springer Science+Business Media, LLC 2012

The introduction of histamine-2 receptor antagonists and, later on, of proton pump inhibitors (PPIs) has revolutionized the treatment of acid-peptic related gastrointestinal disorders. Up to until the 1970s, surgical interventions were considered the best therapeutic options for chronic peptic disorders with highly selective vagotomy being a frequently performed intervention. Even though many patients improved after these interventions, in many cases surgery was not sufficient to relieve symptoms and even resulted in complications, which prompted the development of new therapeutic approaches. Research during the past 40 years into gastric acid related disorders and the development of pharmacological agents that effectively suppress gastric acid stand for a milestone in the management of these diseases. In this respect, PPIs represent a tremendous therapeutic advance, having transformed the lives of patients with previously intractable symptoms of gastro-esophageal reflux and peptic ulcer disease.

Proton pump inhibitors are one of the most frequently prescribed classes of medications worldwide. Global expenditure on these drugs was 10 billion USD in 2006 [1]. A recent socio-demographic study from The Netherlands found that $11.8 \%$ of the population had at least one prescription for a PPI in the year 2006 [2]. In 2010, omeprazole was, together with metoprolol, the most frequently prescribed medication in The Netherlands [3]. The same report described an increase of $24 \%$ in omeprazole prescriptions from 2009 to 2010 , totaling up to 1.2 million prescriptions [3]. The success of this drug class is not only

D. Keszthelyi $(\square) \cdot$ A. A. Masclee

Division of Gastroenterology-Hepatology, Department

of Internal Medicine, Maastricht University Medical Center, Maastricht, The Netherlands

e-mail: daniel.keszthelyi@maastrichtuniversity.nl the result of their potency, unique pharmacokinetics and effectiveness in improving symptoms and complications of acid-peptic disorders, but also the result of their low toxicity. Use of PPIs is considered safe; serious, acute adverse effects are exceedingly uncommon.

However, more recently, a number of concerns have been raised regarding PPI therapy, in particular with respect to long-term use. These include induction of severe hypomagnesemia, iron and vitamin B12 deficiency, communityacquired pneumonia, hip fracture and interstitial nephritis, which have all been found to have a statistically significant association with current and/or long-term PPI use [4]. An extensive discussion in the scientific community has also unfolded regarding a potential increased risk of cardiovascular events when co-administering PPIs with clopidogrel [5]. As far as gastrointestinal conditions are concerned, PPI therapy has also been associated with microscopic colitis (MC) and Clostridium difficile-associated diarrhea. The FDA has in fact recently issued a safety announcement that PPI use may be associated with $C$. difficile infection [6].

Understanding the uncertainties surrounding long-term PPI use is particularly important at present given the general increase in PPI use in recent years, both from the prescription of PPIs by physicians and the self-diagnosis and treatment that has resulted from the over-the-counter availability of the drugs [7]. However, there are few aspects one needs to consider before attempting to ascertain whether an observed statistically significant association between PPI therapy and a certain condition represents a clinically relevant side effect. First, it is inherent to observational studies that the associations found could be attributable to bias and/or confounding factors and not to a true cause-effect relationship. Second, it is important to realize that intervening with gastrointestinal physiology, regardless of its nature (pharmacological or surgical), implies that these interventions, although intended 
as a therapeutic correction, carry the intrinsic potential to cause other effects, even side effects. This also holds true for PPIs. The peculiar issue, however, is that a complete understanding of all the mechanisms and structures affected by PPIs is still lacking, posing an additional obstacle in establishing the certainty of the role of PPIs in inducing such conditions.

The study of $\mathrm{Yu}$ et al. [8], published in this issue of Digestive Diseases and Sciences, adds yet another piece to this puzzle. Yu et al. demonstrate that patients on PPI therapy have significantly higher colonic intraepithelial lymphocyte (IEL) counts compared to patients not exposed to PPIs. In line with this finding, Poullis et al. [9] have demonstrated earlier that fecal calprotectin concentrations are higher in patients on PPI therapy, suggesting a role for PPIs in inducing a mucosal inflammatory reaction. Yu et al. proceed by showing in their study that co-exposure to PPIs and other drugs that have been associated with MC results in an increased risk for elevated IEL counts. An increased IEL count does not necessarily mean that the intestinal mucosa is diseased, it is rather indicative for processes that have the potential to contribute to disease development. The authors indeed proposed that the histological changes observed may potentially represent an early, pre-clinical phase of MC.

The question obviously arises what the origin of such IEL increase might be-a question to which the answer can only be speculative at this point. Amidst all the discussion regarding potential PPI-related risks and side effects, one certainty exists, i.e. that PPIs have the potential to interfere with gastrointestinal physiology by virtue of altering intestinal $\mathrm{pH}$. On of the most profound consequences hereof is an alteration in intestinal microbial profiles [10]. Increase of intestinal $\mathrm{pH}$ can result in a diminished host defense against certain bacteria. The use of PPIs may hence promote the expansion and colonization of pathogenic bacteria potentially resulting in small intestinal bacterial overgrowth (SIBO) [11], although a recent study with over 1900 patients found no significant association between PPI use and SIBO [12]. Therefore, whether changes in intestinal microbiota induced by PPI therapy are truly responsible for the development of symptoms and clinical conditions remains subject to considerable discussion [13].

It is also important to acknowledge that PPIs can also interact with targets other than the gastric $\mathrm{H}^{+} / \mathrm{K}^{+}$ATPase. Colonic epithelial cells also express proton pumps, which are involved in maintaining local electrolyte balance [14]. Several bacteria, including $H$. pylori and S. pneumoniae, as well as fungi such as $C$. albicans, contain $\mathrm{H}^{+} / \mathrm{K}^{+}$ATPase in their plasma membranes which are highly homologous to their human counterparts [7]. PPIs can therefore also influence microbial growth by inhibition of the $\mathrm{H}^{+} / \mathrm{K}^{+}$ATPase.

Proteins other than $\mathrm{H}^{+} / \mathrm{K}^{+}$ATPase have also been reported as targets for PPIs. Omeprazole and lansoprazole have been observed to induce smooth muscle relaxation and to inhibit contractile activity [15]. This effect on contractile systems may also affect tight junction functionality since tight junction proteins are directly linked to the actinomyosin cytoskeleton. Therefore, conformational changes in the cytoskeleton of epithelial cells may result in alterations in the function of the tight junction, which leads to increased paracellular permeability. As a result, luminal contents can more easily penetrate the lamina propria causing an immune and/or inflammatory reaction. Esomeprazole has been shown to increase paracellular permeability in the upper gastrointestinal tract in vitro [16] and in vivo in humans [17]. The intestinal barrier function has been shown to be a key factor in maintaining intestinal homeostasis. Increased paracellular permeability has been observed in a number of gastrointestinal disorders, including MC, and is considered a key component in the induction of diarrhea [18]. Interestingly, elevated numbers of small intestinal bacteria have been linked to significant increases in small intestinal permeability [19], potentially associating these two distinct effects of PPIs.

In an attempt to find an integrative hypothesis, one might postulate that PPIs may induce alterations in intestinal microbiota and/or in intestinal barrier function, albeit not to a clinically significant degree, which can in turn impair the capacity of the intestine to respond to potentially noxious luminal agents. By virtue of interfering with intestinal homeostasis, PPIs may potentially initiate or exacerbate ongoing unfavorable mucosal immune activation, resulting in an increase in IEL count. Such PPIinduced impairment in defense capacity, provided there is a genetic susceptibility present, may therefore lead to the development of a manifest clinical condition (see Fig. 1).

Such a "two-hit" theory conceivably suits the findings of the study by $\mathrm{Yu}$ et al. demonstrating increased risk for higher IEL counts, when PPIs were co-ingested with other drugs or when comorbidities were present. This theory is further supported by a recent animal study by Wallace et al.; administration of omeprazole for 9 days did not cause any inflammation or morpholological change in the jejunal mucosa of rats, but induced significant alterations in the intestinal microbiota. On the other hand, PPIs administration resulted in a marked exacerbation of small intestinal ulceration induced by NSAIDs, which was transferable to germ-free mice via microbiota isolated from the PPI-treated rats, suggesting an important role for microbial alterations [20]. This could provide an explanation for the relevance of co-exposure to PPIs and other drugs in the development of gastrointestinal disorders, including MC.

Certainly, further research will be necessary to test these assumptions and hypotheses, define the exact effects on 


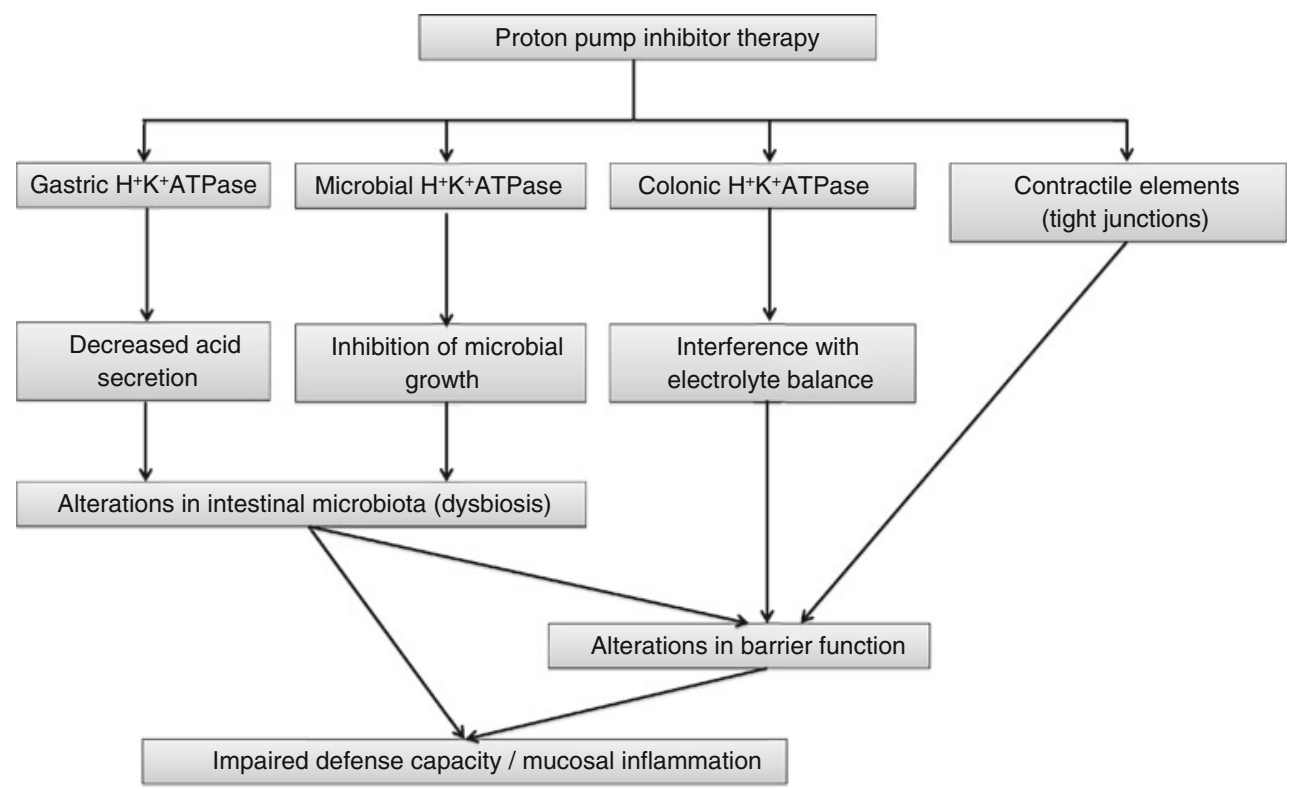

Fig. 1 Schematic representation of postulated mechanisms involved in effects of proton pump inhibitors in the distal gut

PPIs on intestinal physiology and to ascertain whether these changes are of clinical relevance.

\section{Conflict of interest None}

\section{References}

1. Forgacs I, Loganayagam A. Overprescribing proton pump inhibitors. BMJ. 2008;336:2-3.

2. van Boxel OS, Hagenaars MP, Smout AJ, Siersema PD. Sociodemographic factors influence chronic proton pump inhibitor use by a large population in The Netherlands. Aliment Pharmacol Ther. 2009;29:571-579.

3. Stichting Farmaceutische Kerngetallen. Facts and figures 2011, 2012. Available at: http://www.sfk.nl/pdf-documenten/data-en-feiten/dataen-feiten-2011. Accessed July 6, 2012.

4. Ali T, Roberts DN, Tierney WM. Long-term safety concerns with proton pump inhibitors. Am J Med. 2009;122:896-903.

5. Kwok CS, Nijjar RS, Loke YK. Effects of proton pump inhibitors on adverse gastrointestinal events in patients receiving clopidogrel: systematic review and meta-analysis. Drug Saf. 2011;34: $47-57$.

6. Food and Drug Administration Drug Safety Communication. Clostridium difficile-associated diarrhea can be associated with stomach acid drugs known as proton pump inhibitors (PPIs). Available at: http://www.fda.gov/Drugs/DrugSafety/ucm290510. htm. Accessed July 6, 2012.

7. Vesper BJ, Jawdi A, Altman KW, Haines GK III, Tao L, Radosevich JA. The effect of proton pump inhibitors on the human microbiota. Curr Drug Metab. 2009;10:84-89.

8. Yu Y, Han, DS, Choi EY et al. Is use of PPIs related to increased intraepithelial lymphocytes in the colon? Dig Dis Sci. 2012. (Epub ahead of print). doi:10.1007/s10620-012-2315-0.

9. Poullis A, Foster R, Mendall MA, Shreeve D, Wiener K. Proton pump inhibitors are associated with elevation of faecal calprotectin and may affect specificity. Eur $J$ Gastroenterol Hepatol. 2003;15:573-574; author reply 574.

10. Lewis SJ, Franco S, Young G, O'Keefe SJ. Altered bowel function and duodenal bacterial overgrowth in patients treated with omeprazole. Aliment Pharmacol Ther. 1996;10:557-561.

11. Lombardo L, Foti M, Ruggia O, Chiecchio A. Increased incidence of small intestinal bacterial overgrowth during proton pump inhibitor therapy. Clin Gastroenterol Hepatol. 2010;8:504508.

12. Ratuapli SK, Ellington TG, O’Neill MT, et al. Proton pump inhibitor therapy use does not predispose to small intestinal bacterial overgrowth. Am J Gastroenterol. 2012;107:730-735.

13. Spiegel BM. Questioning the bacterial overgrowth hypothesis of irritable bowel syndrome: an epidemiologic and evolutionary perspective. Clin Gastroenterol Hepatol. 2011;9:461-469; quiz e459.

14. Rechkemmer G, Frizzell RA, Halm DR. Active potassium transport across guinea-pig distal colon: action of secretagogues. J Physiol. 1996;493:485-502.

15. Mullin JM, Gabello M, Murray LJ, et al. Proton pump inhibitors: actions and reactions. Drug Discov Today. 2009;14:647-660.

16. Murray LJ, Gabello M, Rudolph DS, et al. Transmucosal gastric leak induced by proton pump inhibitors. Dig Dis Sci. 2009;54: 1408-1417.

17. Mullin JM, Valenzano MC, Whitby M, et al. Esomeprazole induces upper gastrointestinal tract transmucosal permeability increase. Aliment Pharmacol Ther. 2008;28:1317-1325.

18. Burgel N, Bojarski C, Mankertz J, Zeitz M, Fromm M, Schulzke JD. Mechanisms of diarrhea in collagenous colitis. Gastroenterology. 2002;123:433-443.

19. Riordan SM, McIver CJ, Thomas DH, Duncombe VM, Bolin TD, Thomas MC. Luminal bacteria and small-intestinal permeability. Scand J Gastroenterol. 1997;32:556-563.

20. Wallace JL, Syer S, Denou E, et al. Proton pump inhibitors exacerbate NSAID-induced small intestinal injury by inducing dysbiosis. Gastroenterology. 2011;141:1314-1322. 